\title{
Awareness of the Basic Knowledge of Hand Hygiene in Chinese Medical Facilities: A Questionnaire-Based Survey
}

\section{Jing YANG}

Department of infectious diseases, Tianjin Third Central Hospital

\section{Li LONG}

Department of healthcare-associated infection management,Tianjin Third Central Hospital; Extracorporeal life support for critical disease technology research center; Tianjin key laboratory of artificial cell

\section{Shu FENG}

Affiliated Hospital of Zunyi Medical College

\section{Wei SUN}

Graduate Faculty, Tianjin Medical University

Lifang GAO

Tianjin Third Central Hospital

\section{Yamin YANG}

Tianjin Third Central Hospital

Jie LIU

Tianjin Third Central Hospital

\section{Junqing ZHOU}

Tianjin Third Central Hospital

\section{Fuyu ZHANG}

Tianjin Third Central Hospital

Jie TIAN

Tianjin Third Central Hospital

Bin GAO ( $\sim$ gaobinn@yahoo.com )

Tianjin 4th Central Hospital https://orcid.org/0000-0003-3292-7014

\section{Research}

Keywords: China, hand hygiene, basic knowledge, awareness rate

Posted Date: November 4th, 2020

DOI: https://doi.org/10.21203/rs.3.rs-100054/v1

License: (a) (i) This work is licensed under a Creative Commons Attribution 4.0 International License. Read Full License 


\section{Abstract}

Background: Hand hygiene is a key measure for the prevention and control of healthcare-associated infection. We investigated the level of awareness of the basic concepts surrounding hand hygiene among healthcare staff in China in order to inform the development of future targeted hand hygiene promotion activities.

Methods: A pre-tested questionnaire was used to obtain data from April to June 2018 in 30 provinces and municipalities in three regions of China. Ten single/multiple-choice questions investigated the level of awareness of the role of hands in germ transmission, the concept of hand hygiene, and the indications when it should be performed. Logistic analysis was performed to identify differences between regions, types of hospitals, healthcare staff categories, clinical departments and gender.

Results: A total of 52,286 responses were received. Among these, 45,455 (86.94\%) complete surveys were eligible to be included in the final analysis. The two highest correct response rates concerned the relationship between hand hygiene and healthcare-associated infection and the characteristics of microbiota and resident flora on hands ( $97.71 \%$ and $96.35 \%$, respectively). These were followed by awareness of the World Health Organization's "My 5 moments for hand hygiene" concept (91.61\%), glove use (90.53\%), hand hygiene materials (89.28\%), characteristics of microbiota and transient flora on hands (84.60\%), indications for hand hygiene and other aspects (71.00\%), the basic concept of hand hygiene (65.20\%), and the effectiveness of alcohol-based handrub on the hand microbiome (48.04\%). The overall correct response rate was $26.53 \%$. Eastern China demonstrated a better knowledge than other regions (adjusted odds ratio [aOR] 4.08; 95\% Cl 3.84-4.32). Respondents in primary care facilities had a higher correct response rate than other types of hospitals (aOR $2.33 ; 95 \% \mathrm{Cl} 2.05-2.66)$ and logistics staff had the highest correct response rate than other professional groups (aOR1.84; 95\% Cl 1.532.29). Among clinical departments, staff in the outpatient/emergency room had the highest rate of correct responses (aOR1.51; 95\% 1.341.71).

Conclusions: Large differences exist in the basic knowledge of hand hygiene across different regions in China. Knowledge levels need to be specifically strengthened among secondary care facilities, nurse professionals, gynaecology/obstetrics/paediatric departments and infection control units.

\section{Introduction}

Hand hygiene is recognized worldwide as an important element of hospital infection control [1,2]. As early as 2009, the Ministry of Health of the People's Republic of China issued "Standards for hand hygiene for healthcare workers in healthcare settings (CSHH/WS/T 1312009)" to be strictly enforced and implemented nationwide[3]. However, no formal assessment of their uptake has been undertaken, apart from one study of secondary and tertiary hospitals in 14 provinces, mainly focused on knowledge of the World Health Organization (WHO) "My 5 moments for hand hygiene" concept [4].Our main study objective was to investigate the current level of basic knowledge of hand hygiene across a wide range of healthcare facilities and regions in China in order to help inform a national strategy to promote hand hygiene.

\section{Methods}

\section{Survey recipients}

We conducted a questionnaire-based survey between April and June 2018 focused on the basic knowledge of hand hygiene among healthcare workers in primary, secondary and tertiary hospitals in the three main regions of Mainland China as defined by the Almanac of Health Statistics of China [5], i.e., East(Beijing, Tianjin, Hebei, Liaoning, Shanghai, Jiangsu, Zhejiang, Fujian, Shandong, Hainan, and Guangdong), Central (Shanxi, Henan, Heilongjiang, Jilin, Anhui, Jiangxi, Hubei, and Hunan)and West (Inner Mongolia, Chongqing, Guangxi, Guizhou, Sichuan, Yunnan, Xizang, Gansu, Ningxia, Qinghai, Shaanxi, and Xinjiang). Ethics approval was granted by the ethics committee of the Third Central Hospital of Tianjin(no.: IRB2018-032). The anonymity of respondents was optional.

The survey comprised 10 single/multiple-choice questions based on the CSHH concerning knowledge of the following: individual awareness of the concept of hand hygiene and transmission of pathogens by hands; the relationship between hand hygiene and healthcare-associated infection; appropriate hand hygiene materials; the WHO "My 5 moments for hand hygiene" concept; indications and knowledge of other aspects of hand hygiene practices, such as glove use; and the effectiveness of alcohol-based handrub for the rapid elimination of transient skin flora and reduction of resident flora(Figure; Additional file 1).

\section{Questionnaire distribution and data collection}


Questionnaires were distributed to healthcare workers recruited using the snowball sampling method. A social media platform (BlueDragon Network) was used for data collection. This platform is an online survey application (app) that facilitates the distribution of questionnaires by e-mail or smartphones by using messaging apps, such as WeChat and WhatsApp. It allows participants to access the questionnaire easily and has the capacity to analyze and export results after responses have been collected[6]. Data on respondents' hospital location and type of facility, gender and professional status were also collected. Hospitals were classified according to the Chinese National Healthcare Institutes' Enquiry System (http://zgcx.nhc.gov.cn:9090/unit/index) or their site homepage if not registered in the Enquiry System. Participation did not involve any financial compensation. No confidential data were collected and no reminders to participate were sent. Questionnaires without accurate hospital names were excluded, as well as those with incomplete data. When the user name and workplace was used more than once, only the first questionnaire was retained.

\section{Statistical analysis}

Survey responses were downloaded in Excel format. Data were digitally stored and analyzed using SPSS, version 17 (SAS Institute Inc, Cary, NC, USA). Categorical and continuous variables were described as numbers (\%). Descriptive statistics were used as appropriate. Univariate and multivariate binary logistic regression analysis was performed for each independent variable associated with an awareness of hand hygiene. A $P$-value of less than 0.05 was considered statistically significant for all analyses.

\section{Results}

\section{Demographic characteristics of respondents}

A total of 52,286 questionnaires were returned. Of these, 6,305 duplicates were deleted. A further 526 were deleted as the workplace was unable to be ascertained, and the location and type of facility were rectified in 5,445 . The final analysis included 45,455 eligible questionnaires from 961 hospitals across 30 provinces/municipalities. Respondents were physicians (12,353[27.18\%]), nurses (26,290 [57.84\%]), medical technicians (4,415 [9.71\%]), administrative staff (1,785 [3.93\%]) and logistics personnel (612 [1.35\%]); 37,490 (82.84\%) were female. Workplace locations included paediatric units, gynaecology and obstetric departments, surgical units, internal medicine, outpatient/emergency room departments, medical-technical departments, administration-logistics, as well as infection control units (Table 1). 
Table 1

Characteristics of responding participants.

\begin{tabular}{|c|c|c|c|c|c|}
\hline & $\begin{array}{l}\text { Non-registeredhospital } \\
(\mathrm{N}=82)\end{array}$ & $\begin{array}{l}\text { Primary hospital } \\
(\mathrm{N}=82)\end{array}$ & $\begin{array}{l}\text { Secondary hospital } \\
(\mathrm{N}=498)\end{array}$ & $\begin{array}{l}\text { Tertiary hospital } \\
(\mathrm{N}=299)\end{array}$ & $\begin{array}{l}\text { Total } \\
(\mathrm{N}=961)\end{array}$ \\
\hline \multicolumn{6}{|l|}{ Male gender $n(\%)$} \\
\hline - Total & $148(12.18)$ & 194(17.21) & $2637(17.34)$ & 4986(17.87) & 7965(17.52) \\
\hline - Physician & $83(26.10)$ & $119(28.61)$ & $1826(43.12)$ & $3293(44.60)$ & $5321(43.07)$ \\
\hline - Nurse & $8(1.43)$ & $0(0.00)$ & 139(1.64) & $561(3.32)$ & $708(2.69)$ \\
\hline - Administrative staff & 21(13.13) & $22(15.49)$ & $100(13.21)$ & $121(16.67)$ & 264(14.79) \\
\hline - Medical-technical staff & $28(20.14)$ & $47(25.13)$ & $482(31.75)$ & $876(34.07)$ & 1433(32.46) \\
\hline - Logistical personnel & $8(20.51)$ & $6(30.00)$ & $90(44.78)$ & 135(38.35) & 239(39.05) \\
\hline \multicolumn{6}{|l|}{ Work unit n(\%) } \\
\hline - Medicine & 232(19.09) & $440(39.04)$ & $4840(31.83)$ & 10435(37.39) & 15947(35.08) \\
\hline - Surgery & 165(13.58) & $87(7.72)$ & $3179(20.90)$ & $6934(24.85)$ & $10365(22.80)$ \\
\hline - Gynaecology-obstetrics & 211(17.37) & $61(5.41)$ & $1766(11.61)$ & $1870(6.70)$ & $3908(8.60)$ \\
\hline - Paediatric unit & $129(10.62)$ & 12(1.06) & 1168(7.68) & $1704(6.11)$ & $3013(6.63)$ \\
\hline - Outpatient-emergency & $91(7.49)$ & $75(6.65)$ & $1028(6.76)$ & 1999(7.16) & $3193(7.02)$ \\
\hline - Medical-technical units & $154(12.67)$ & $217(19.25)$ & $2076(13.65)$ & $3679(13.18)$ & 6126(13.48) \\
\hline - Administration-logistics & 199(16.38) & $213(18.90)$ & $725(4.77)$ & $926(3.32)$ & 2063(4.54) \\
\hline - Infection control unit & $34(2.80)$ & $22(1.95)$ & $425(2.79)$ & $359(1.29)$ & $840(1.85)$ \\
\hline \multicolumn{6}{|l|}{ Geographic region(\%) } \\
\hline - Eastern China & $432(35.56)$ & $987(87.58)$ & $4202(27.63)$ & $11640(41.71)$ & 17261(37.97) \\
\hline - Central China & $370(30.45)$ & $90(7.99)$ & $5605(36,86)$ & 9313(33.37) & 15378(33.83) \\
\hline - Western China & 413(33.99) & $50(4.44)$ & $5400(35.51)$ & 6953(24.92) & $12816(28.19)$ \\
\hline
\end{tabular}

\section{Awareness level of the concept of hand hygiene}

The average accuracy rate of all questions was $81.55 \%$. Overall, the highest correct response rate $(97.71 \%)$ was to question 4 (relationship between hand hygiene and healthcare-associated infection), closely followed by question 1 (transmission of pathogens by hands[96.35\%]). The correct response rate to questions10, 8 and 7 ("My 5moments for hand hygiene" concept, glove use and hand hygiene, and knowledge of hand hygiene materials) were relatively good $(91.61 \%, 90.53 \%$, and $89.28 \%$, respectively). The correct response rate to questions 6 and 2 (transmission of pathogens by hands and the effect of alcohol-based handrub on the total number of bacterial colonies present) were generally average ( $84.60 \%$ and $81.21 \%$, respectively). The correct response rate to questions 9 and 5 (indications and knowledge of other aspects of hand hygiene practices and the concept of hand hygiene) were less than average $(71.00 \%$ and $65.20 \%$, respectively). The correct response rate to question 3 (effectiveness of alcohol-based handrub on transient skin flora) was generally poor (48.04\%). The 10/10 correct rate was $26.53 \%(P<0.05)$ (Fig. 1).

\section{Awareness level of the basic knowledge of hand hygiene by type of healthcare worker and hospital facility}

The linear regression model showed that the following healthcare worker characteristics were associated with a statistically significant overall accuracy of responses: male gender; professional status related to logistics/administration or a medical technician/physician; working in an outpatient/emergency unit, surgical or medical technical unit, internal medicine service, and administration/logistics; located in a primary, secondary or tertiary care hospital or a non-classified hospital (i.e., medical facility not registered in the Chinese National Healthcare Institute's Enquiry System); and located in Eastern and Central China(Table 2). 
Table 2

Factors associated with 10/10knowledge scores of hand hygiene.

\begin{tabular}{|c|c|c|c|}
\hline & Responses with a10/10 score (n [\%]) & $\begin{array}{l}\text { Univariate analysis } \\
\text { (OR [95\% Cl]) }\end{array}$ & $\begin{array}{l}\text { Multivariate analysis } \\
\text { (adjusted OR [95\% Cl]) }\end{array}$ \\
\hline \multicolumn{4}{|l|}{ Hospital rating } \\
\hline -Non-registered hospital & $398(32.76 \%)$ & $1.89(1.66,2.14)$ & $1.75(1.53,1.99)$ \\
\hline -Primary hospital & $635(56.34 \%)$ & $5.00(4.41,5.66)$ & $2.33(2.05,2.66)$ \\
\hline -Secondary hospital & $3,122(20.54 \%)$ & 1.00 & 1.00 \\
\hline -Tertiary hospital & $7,902(28.32 \%)$ & $1.53(1.46,1.60)$ & $1.28(1.22,1.34)$ \\
\hline \multicolumn{4}{|l|}{ Geographic region } \\
\hline -Eastern China & $7,356(42.62 \%)$ & $4.36(4.11,4.62)$ & $4.08(3.84,4.32)$ \\
\hline -Central China & $2,835(18.44 \%)$ & $1.33(1.24,1.41)$ & $1.36(1.27,1.45)$ \\
\hline -Western China & $1,866(14.56 \%)$ & 1.00 & 1.00 \\
\hline \multicolumn{4}{|l|}{ Gender } \\
\hline -Female & $9,757(26.03 \%)$ & 1.00 & 1.00 \\
\hline -Male & $2,300(28.88 \%)$ & $1.15(1.09,1.22)$ & $0.96(0.90,1.02)$ \\
\hline \multicolumn{4}{|l|}{ Professional status } \\
\hline - Physician & $3,427(27.74 \%)$ & $1.23(1.17,1.29)$ & $1.22(1.15,1.29)$ \\
\hline - Nurse & $6,248(23.77 \%)$ & 1.00 & 1.00 \\
\hline - Administrative staff & $569(31.88 \%)$ & $1.50(1.35,1.67)$ & $1.65(1.41,1.94)$ \\
\hline - Medical-technical staff & $1,544(34.97 \%)$ & $1.73(1.61,1.85)$ & $1.61(1.46,1.78)$ \\
\hline - Logistics staff & $269(43.95 \%)$ & $2.52(2.14,2.96)$ & $1.84(1.53,2.29)$ \\
\hline \multicolumn{4}{|l|}{ Work unit } \\
\hline - Paediatric unit & $597(19.81 \%)$ & 1.00 & 1.00 \\
\hline - Gynaecology-obstetrics & $822(21.03 \%)$ & $1.08(0.96,1.21)$ & $1.07(0.95,1.21)$ \\
\hline - Infection control unit & $186(22.14 \%)$ & $1.15(0.96,1.39)$ & $0.86(0.69,1.11)$ \\
\hline - Outpatient-emergency & $886(27.75 \%)$ & $1.55(1.38,1.75)$ & $1.51(1.34,1.71)$ \\
\hline - Medicine & $4,095(25.68 \%)$ & $1.40(1.27,1.54)$ & $1.33(1.20,1.47)$ \\
\hline - Surgery & $2,733(26.37 \%)$ & $1.45(1.31,1.60)$ & $1.44(1.30,1.60)$ \\
\hline - Medical-technical units & $1,980(32.32 \%)$ & $1.93(1.74,2.15)$ & $1.40(1.23,1.58)$ \\
\hline - Administration-logistics & $758(36.74 \%)$ & $2.35(2.07,2.67)$ & $1.28(1.08,1.51)$ \\
\hline Total & $12,057(26.53 \%)$ & & \\
\hline
\end{tabular}

Results of the multivariate logistic regression analysis, with the outcome variable of full-score marks (10/10), also confirmed that the odds of an appropriate awareness of hand hygiene were higher among the following categories: healthcare workers working in logistics (adjusted odds ratio [aOR]1.84; 95\% confidence interval [Cl] 1.53-2.29), administration (aOR 1.65; 95\% Cl 1.41-1.94)and as a medical technician (aOR 1.61;95\% Cl1.46-1.78) and physician (aOR 1.22; 95\% Cl1.15-1.29). The overall accuracy of respondents was also more likely if healthcare workers worked in an outpatient/emergency unit (aOR 1.51; 95\% $\mathrm{Cl} 1.34-1.71$ ), surgical department (aOR 1.44; 95\% Cl 1.30-1.60), medical-technical unit (aOR 1.40; 95\% Cl 1.23-1.58), internal medicine department (aOR 1.33; 95\% Cl 1.20-1.47) and administration-logistics (1.28; 95\% Cl 1.08-1.51). Similarly, working in a primary care hospital (aOR 2.33; 95\% Cl 2.05-2.66), nonregistered hospital $(1.75 ; 95 \% \mathrm{Cl} 1.53-1.99)$ and a tertiary care hospital (aOR1.28; 95\% Cl 1.22-1.34) located in Eastern (aOR 4.08; 95\% Cl 3.84-4.32) and Central China (aOR 1.36; 95\% Cl 1.27-1.45) were all associated with a higher rate of accurate responses(Table 2). 


\section{Discussion}

The global antimicrobial drug resistance situation remains extremely preoccupying and transmission by the hands of healthcare workers has been identified as a major cause of the rapid increase of multidrug-resistant bacteria[7]. Several studies have confirmed that improving the hand hygiene compliance rate among healthcare workers can effectively reduce the incidence of healthcare-associated infection $[8,9]$. In addition, in the context of the current pandemic situation related to the spread of the new coronavirus (SARS-CoV-2), hand hygiene is a key infection prevention measure to help combat transmission both in the healthcare setting and in the community[10]. Since the legal requirement concerning hand hygiene standards in China was instituted in 2009, as well as the continuous development of hand hygiene promotion over the past decade, compliance with appropriate hand hygiene practices in medical facilities has gradually increased[11], but still remains suboptimal[12, 13], similar to other countries worldwide[14].

We consider that our survey results comprising 45,455 healthcare workers in the main three regions of China can be considered as a true reflection of the awareness of hand hygiene basic knowledge in the country to a large extent. These findings also provide national health authorities with valuable scientific data for the development of further targeted training on hand hygiene to improve the implementation rate of recommendations within facilities. The average correct response rate to all 10 questions was $81.55 \%$. However, the overall accuracy of respondents to all 10 questions was only $26.53 \%$.

The correct response rate to the question related to the knowledge and understanding of the "My 5 moments for hand hygiene" concept revealed an accuracy rate above $91.96 \%$, although the results of the earlier national hand hygiene survey reported by $\mathrm{Xu}$ and colleagues were slightly lower for this specific hand hygiene knowledge point[4]. The misunderstanding related to this question may be that many healthcare workers believed that wearing gloves can replace hand hygiene. Indeed, the review of Sun and Gao highlighted that several studies revealed that appropriate hand hygiene practices had been replaced by glove use in China[15] and this erroneous notion must be corrected rapidly. Although the responses to questions 10, 8 and 7 ("My 5 moments for hand hygiene" concept, glove use and hand hygiene, and knowledge of materials used for hand hygiene) were approximately $90 \%$, the question related to the indications and knowledge of other aspects of hand hygiene practices was relatively low $(71.00 \%)$.

The highest correct response rate (96.25\%) concerned the relationship between hand hygiene and the acquisition of healthcare-associated infection, whereas the response investigating awareness related to the actual concept of hand hygiene was only $65.20 \%$. Another inconsistency of awareness related to microbiota on hands. The correct response to the question on the characteristics of resident flora on hands was high (96.35\%). However, the correct response concerning the characteristics of transient flora on hands was only $84.60 \%$, and the response concerning the effectiveness of alcohol-based handrub on transient flora even lower (81.21\%). Only a mere $48.04 \%$ of participants responded correctly to the question on the effect of handrubbing on the number of microbiota on hands. Therefore, this suggests that a more in-depth evaluation is needed to understand and address the factors that have impeded an awareness of these notions.

Logistic analysis showed that the correct response rate was higher in Eastern and Central China than Western regions. In 2018, the number of healthcare workers per 1000 population in the Eastern, Central and Western regions of China was 7.2, 6.2 and 6.9, respectively, and the hospitalization rate of the population in 2018 was $16.5 \%, 18.7 \%$ and $20.3 \%$, respectively[5]. Based on these data, differences of awareness of the importance of hand hygiene may help to act as a guide to drive improvement at the hospital administrative level, particularly in the Western region.

Our analysis of the basic knowledge of hand hygiene in different types of hospitals suggested that staff awareness was best in primary care hospitals, followed by non-registered and tertiary care hospitals, while awareness in secondary facilities were poor. This is unexpected and inconsistent with the research by Shen and colleagues[13] and slightly different from the results of Xu and colleagues and Qin and colleagues $[4,12]$. This may be related to the number and scope of knowledge points on hand hygiene addressed in our questionnaire. Our survey results showed that primary care hospitals have been attentive to the spread of hand hygiene knowledge in recent years and, consequently, the awareness rate has greatly improved. Tertiary hospitals have more training opportunities, stricter management and a stronger sense of the importance of hand hygiene. The weakest link lies in secondary hospitals where training is not in place and the emphasis on prevention measures for healthcare-associated infection is insufficient. This must be the key target for hand hygiene education in the future in China.

Knowledge of hand hygiene was poorest among nurses compared to other healthcare categories. Interestingly, the awareness level of logistics personnel was greatly improved than previously observed, thus indicating that medical facilities have carried out effective strategies across all categories of healthcare workers to improve the weak medical knowledge of this profession in recent years. Although nurses and physicians have the most clinical interaction with patients, some aspects of their knowledge of the basic concepts surrounding 
hand hygiene were sadly lacking. This was unexpected and did not correlate with the previous reports by Deng and colleagues and Xiao and colleagues[16, 17] In terms of microbial knowledge of hand flora and correct hand hygiene practices[17], knowledge was rather poor, especially concerning the use of sterile gloves to replace hand hygiene, as previously reported[18].

Healthcare workers in the outpatient/emergency room performed best among the different departments, while those in gynaecology/obstetrics, paediatrics and infection control units performed poorly. For the gynaecology/obstetric departments where more clinical procedures occur, hand hygiene should be the focus of a specific intervention and awareness-raising strategy. It is difficult to accept that infection control personnel were less knowledgeable, but this has already been documented elsewhere [19]. Our data suggest also that it remains necessary to continue to strengthen hand hygiene training for infection prevention and control staff by developing an occupational license or a professional development programme integrated into the regular educational activities of the various departments, notably for clinical frontline medical staff. In particular, infection prevention and control services in hospitals need to heighten their profile with the full and visible support of the directorate in order to make all staff aware of the importance of their work and its translation into real practice. WHO has proposed "Clean Care is Safer Care" as a priority initiative to improve medical quality in the $21 \mathrm{st}$ century [20]. However, this cannot be achieved if major efforts are not made to continue to improve the compliance of medical staff with optimal practices.

\section{Limitations}

Our study has some limitations. First, the number of participants in the different regions, as well as the types of hospital, healthcare worker categories, gender and clinical departments, is not homogenous. Second, in terms of the study design, there are some limitations, such as a lack of investigation of some aspects of hand hygiene, e.g. surgical hand antisepsis and knowledge of multimodal intervention strategies for hand hygiene. Third, there was heterogeneity across the different regions regarding the types of hospital and staff categories (e.g. educational background, length of service) with less primary care hospitals involved, particularly in Central and Western China. Finally, the specific issue related to the limited duration of the shelf life of handrub/gel has been updated in the new edition of the $\mathrm{CSHH}$ (WS/T 313-2019)[21], and all other points of awareness in our questionnaire will also be included in the updated edition.

\section{Conclusions}

Large differences exist in the basic knowledge of hand hygiene in healthcare facilities in different regions in China. In particular, it is important to strengthen knowledge in medical facilities of some Western provinces. Basic knowledge also needs to be more extensively promoted in secondary hospitals and among nurses and staff working in gynaecology/obstetrics and paediatric departments, and particularly in infection prevention and control units.

\section{Abbreviations}

CSHH: China-issued Standard for hand hygiene for healthcare workers in healthcare settings.

WHO: World Health Organization

\section{Declarations}

\section{Acknowledgements}

We thank Rosemary Sudan for providing editorial assistance with earlier drafts of the document and her indispensable support.

\section{Authors' contributions}

LL, JY, FZ and TJ were involved in the study design and implementation. LL, LG, YY, JL and JZ undertook data collection. LL, SF, WS and BG performed the data analysis and designed the figures and tables. SF, WS and BG were involved in data interpretation and study coordination. JY and LL wrote the manuscript.BG reviewed the manuscript. All authors have read and approved the final version for publication.

\section{Funding}

Supported by the Tianjin Municipal Health Planning Commission Self-funded Fund Project (2014KZ012). 
Availability of supporting data

All the research data are reliable and available upon request to the corresponding author.

\section{Ethical approval and consent to participate}

The study was approved by the ethics committee of the Third Central Hospital of Tianjin (IRB2018-032).

\section{Consent for publication}

The material is original and has not been published. All authors have seen and agreed to submit the final version of the paper.

\section{Competing interests}

The authors declare that they have no conflict of interest.

\section{References}

1. Mukerji A, Narciso J, Moore C, McGeer A, Kelly E, Shah V. An observational study of the hand hygiene initiative: a comparison of preintervention and postintervention outcomes. BMJ Open 2013;3(5).

2. World Health Organization. WHO Guidelines on hand hygiene in health care. Geneva, Switzerland: WHO; 2009.https://www.who.int/publications/i/item/who-guidelines-on-hand-hygiene-in-health-care. Accessed 20 October 2020.

3. Standards for hand hygiene for healthcare workers in hospital settings. WS/T 313-2009. Beijing: National Health Commission of the People's Republic of China. https://www.chinesestandard.net/PDF/English.aspx/WST313-2019. Accessed 20 October 2020.

4. Xu D, Hou T, Lei W, Ma Q, Yang H, Ding L, et al. Awareness of hand hygiene knowledge and compliance status in Chinese hospitals. Chinese J Infect Control 2016;9:654-658, 664.

5. National Health Commission of the People's Republic of China. China health statistics almanac 2019. Beijing: Peking Union Medical College Press; 2019

6. Kingston LM, Slevin BL, O'Connell NH, Dunne CP. Hand hygiene: attitudes and practices of nurses, a comparison between 2007 and 2015. Am J Infect Control 2017;45:1300-1307.

7. Kingston LM, O'Connell NH, Dunne CP. Survey of attitudes and practices of Irish nursing students towards hand hygiene, including handrubbing with alcohol-based hand rub. Nurse Educ Today 2017;52:57-62.

8. Bukhari SZ, Hussain WM, Banjar A, Almaimani WH, Karima TM,Fatani MI. Hand hygiene compliance rate among healthcare professionals. Saudi Med J 2011;32:515-519.

9. De Wandel D, Maes L, Labeau S, Vereecken C, Blot S. Behavioral determinants of hand hygiene compliance in intensive care units. Am J Crit Care 2010;19:230-239.

10. Tartari E, Hopman J, Allegranzi B, Gao B, Widmer A, Cheng VC, et al. Perceived challenges of COVID-19 infection prevention and control preparedness: amultinational survey. J Glob Antimicrob Resist 2020;22:779-781.

11. Zhao S, Fu Q. Status of healthcare-associated infection management in private medical institutions in China. Chin J Infect Control. Chin J Infect Control 2018;17:391-394.

12. Xin Q, Yu L, Wei J, Wang P, Li J, Li H, et al. Current situation of hand hygiene of medical staff in 18 medical institutions. Chinese J Nosocomiology 2017;8:1905-1908.

13. Shen Y, Hu B, Zhou Q, Gao X, Cui Y, Sun W. Current status of hand hygiene compliance of 66 hospitals in Shanghai. Chinese J Nosocomiology 2012;12:2585-2587.

14. Tartari E, Pires D, Bellissimo-Rodrigues F, De Kraker M, Borzyowski TH, Allegranzi B, et al. The global hand-sanitizing relay: promoting hand hygiene through innovation. J Hosp Infect 2017;95:189-193.

15. Sun Y, Gao B. Existing problems and countermeasures in clinical application of medical gloves. Chin J Infect Control 2018;17:10-940944.

16. Deng S, Deng J, Zhang J. Investigation on hand contamination among staff in a grass-roots hospital. Chinese J Disinfection 2016;1:69-71.

17. Xiao J, Fan Z, Mi X, Li L, Lin L. A comparative study on hand hygiene knowledge, attitude and behavior of medical staff from different level medical institutions. Chinese J Disinfection 2018;5:335-338. 
18. Wang L. Current status of hand hygiene in clinical staff. Chinese J Disinfection 2011;4:488-489.

19. Yang Y, Zhang H, Hu L, Cai L, Zhang Y, Zhang X, et al. Hand hygiene cognition among healthcare-associated infection management staff in Gansu province. Chin J Infect Control 2019;2:142-146.

20. Tartari E, Abbas M, Pires D, De Kraker M, Pittet D. World Health Organization SAVE LIVES: Clean Your Hands global campaign-'Fight antibiotic resistance-it's in your hands'. Clin Microbiol Infect 2017;23:596-598.

21. Specification of hand hygiene for healthcareworkers.Beijing: National Health Commission of the People's Republic of China; 2019. http://www.nhc.gov.cn/wjw/s9496/202002/dbd143c44abd4de8b59a235feef7d75e/files/6a3e2bf3d82b4ee8a718dbfc3cde8338.pdf. Accessed 20 October 2020.

\section{Figures}

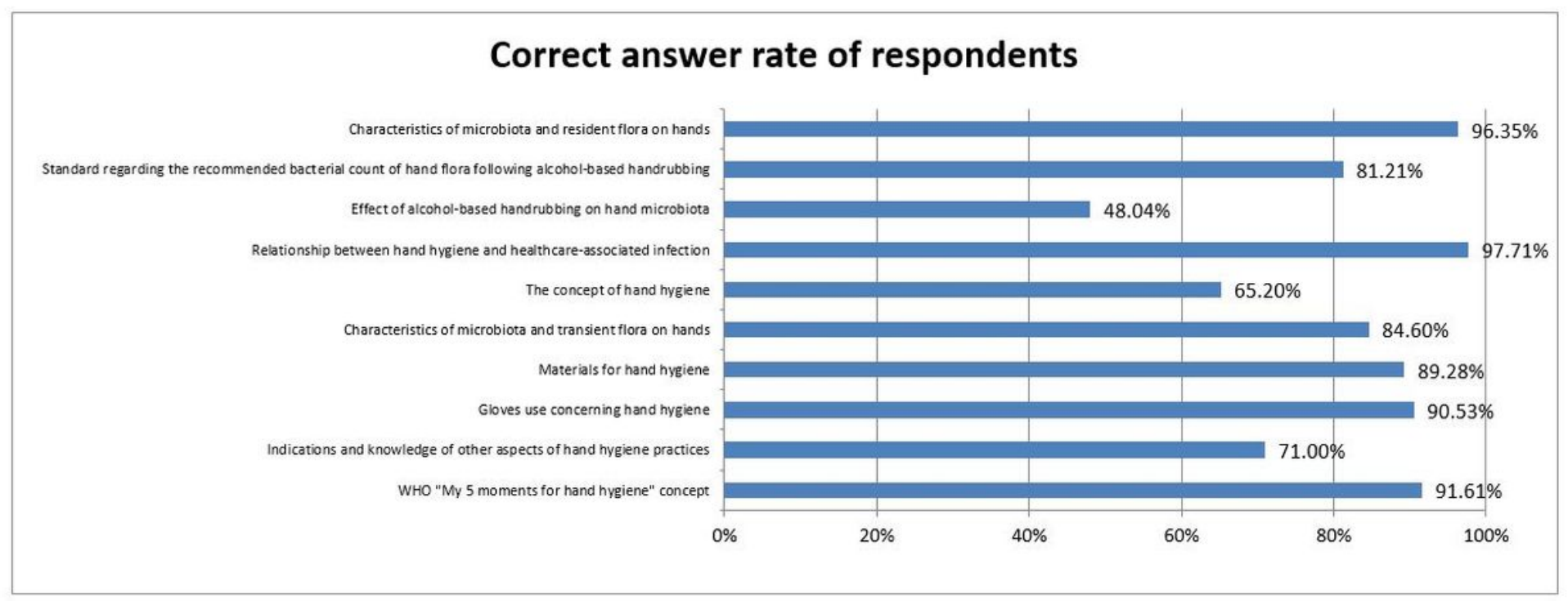

\section{Figure 1}

Correct answer rate of respondents according to the questionnaire themes $(n=45,455)$.

\section{Supplementary Files}

This is a list of supplementary files associated with this preprint. Click to download.

- ARICQuestionnaire.pdf 